\title{
Mercado de suelo en ciudades petroleras. Barrancabermeja (Colombia) y Comodoro Rivadavia (Argentina), 2000-2018
}

\author{
Mercado de terras em cidades petrolíferas. Barrancabermeja (Colômbia) e Comodoro \\ Rivadavia (Argentina), 2000-2018
}

Resumen: En el presente artículo, analizamos el mercado de suelo en dos ciudades petroleras de América Latina en medio de las fuertes fluctuaciones del precio del petróleo en el siglo XXI. Pretende ser un primer acercamiento cualitativo a su funcionamiento y una primera comparación entre las dos ciudades. Las fuentes para el trabajo son esencialmente bibliográficas, aunque se incluyen algunas entrevistas realizadas a referentes del mercado inmobiliario y documentos estadísticos que dan información sobre la construcción.
Resumo: Neste artigo, analisamos o mercado de terras em duas cidades petrolíferas da América Latina em meio às fortes oscilações do preço do petróleo no século XXI. Pretende-se ser uma primeira abordagem qualitativa ao seu funcionamento e uma primeira comparação entre as duas cidades. As fontes do trabalho são essencialmente bibliográficas, embora incluam algumas entrevistas com líderes do mercado imobiliário e documentos estatísticos que fornecem informações sobre a construção.

\section{Javier Serrano Besil}

Doctorando en Ciencias Sociales, Facultad de Ciencias Sociales - Universidad de Buenos Aires

Instituto de Investigaciones Gino Germani - CONICET

Dirección: Av. Santa Fe 2338

Teléfono: +54 91139474690

E-mail: jserranobesil@gmail.com 


\title{
Mercado de suelo en ciudades petroleras. Barrancabermeja (Colombia) y Comodoro Rivadavia (Argentina), 2000-2018
}

\author{
Real Estate Market in Oil Cities. Barrancabermeja (Colombia) and Comodoro Rivadavia
}

(Argentina), 2000-2018

\begin{abstract}
In this article, we analyze the land market in two oil cities in Latin America amidst the strong fluctuations in the price of oil in the 21 st century. It is intended to be a first qualitative approach to its operation and a first comparison between the two cities. The sources for the work are essentially bibliographic, although some interviews with real estate market leaders and statistical documents that provide information on construction are included.
\end{abstract}

\author{
Javier Serrano Besil \\ Doctorando en Ciencias Sociales, Facultad de Ciencias Sociales - Universidad de Buenos Aires \\ Instituto de Investigaciones Gino Germani - CONICET \\ Dirección: Av. Santa Fe 2338 \\ Teléfono: +54 91139474690 \\ E-mail: jserranobesil@gmail.com
}




\section{Introducción}

La historia, el crecimiento y la configuración socioespacial de Barrancabermeja (Colombia) y Comodoro Rivadavia (Argentina) están relacionados al descubrimiento y explotación de petróleo, y, por tanto, al mercado internacional que establece el precio del barril de crudo. Tras su establecimiento a inicios del siglo XX, la industria petrolera rápidamente se impuso en las dos ciudades como la actividad económica principal. En un movimiento contradictorio, relegó a la incipiente actividad comercial y agrícola previa a ser subsidiaria y complementaria de las necesidades petroleras al mismo tiempo que dinamizó e impulsó su crecimiento.

En el caso colombiano, en 1916, el gobierno nacional entregó, en concesión a la Tropical Oil Company (filial de la Standard Oil, de Nueva Jersey, EE.UU), el territorio del yacimiento para extraer y refinar el crudo de la zona. La empresa norteamericana logró desembarcar luego de algunas dificultades políticas', pero, tras el inicio oficial de los trabajos, incrementó rápidamente los niveles de producción. La ciudad se transformó. Una fragmentación inicial del espacio se evidenció como consecuencia del establecimiento de un enclave extranjero: por un lado, aparecía la "Barrancabermeja gringa", que contrastaba en sus espacios y funciones con una "Barrancabermeja colombiana". Entre tanto, en Comodoro Rivadavia, un día después del hallazgo del yacimiento en 1907, se firmó la reserva estatal para la explotación del petróleo encontrado tres kilómetros al norte del pueblo fundado tan solo seis años atrás. A pesar de la decisión de restringir los cateos y trabajos en la zona, varias empresas privadas exploraron y explotaron el territorio colindante a la reserva estatal. Este proceso fue el disparador de una mayor fragmentación territorial. Cada empresa que lograba extraer con éxito petróleo creaba un campamento que

$1 \quad$ Las relaciones entre Colombia y Estados Unidos se vieron afectadas por la intervención norteamericana en la independencia de Panamá. Palacios (1991) argumenta que la entrega final del yacimiento está relacionada con el pago de una indemnización que terminó por sacar "la espina de Panamá" (Palacios, 1995). funcionaba como pueblo autónomo, configurando una multipolaridad urbana notoria.

En la actualidad, Barrancabermeja y Comodoro Rivadavia son ciudades intermedias, que, en cifras oficiales, están rondando los doscientos mil habitantes, aunque, en el caso de Comodoro Rivadavia, algunos relevamientos locales señalan que la población real de la ciudad podría alcanzar los trescientos mil habitantes (Barrionuevo, 2016)². La alta movilidad poblacional asociada a la actividad petrolera y las oscilaciones en el precio del barril y las agendas de inversiones inciden en la actividad comercial y constructiva. En los dos casos, el mercado inmobiliario experimentó cambios repentinos y procesos cíclicos marcados por las fluctuaciones petroleras. En el siglo XXI, el valor del petróleo tuvo oscilaciones significativas. Los precios alcanzaron máximos y mínimos históricos, haciendo difícil prever las condiciones en el largo plazo: "entre mediados del año 2008 cuando llegó alrededor de USD 140 por barril y su posterior caída a menos de USD 40 por barril a principios de 2009 debido a la crisis financiera, pasando por una paulatina pero sostenida recuperación hasta llegar a más de USD 100 por barril en junio de 2014 y nuevamente caer a la mitad a partir de inicio del año 2015" (Arroyo y Cosío, 2015).

El objetivo del presente trabajo es analizar el mercado inmobiliario de Barrancabermeja y Comodoro Rivadavia durante lo que va corrido del siglo XXI. Pretende ser un primer acercamiento cualitativo a su funcionamiento y una primera comparación entre las dos ciudades. Las fuentes para el trabajo son esencialmente bibliográficas, aunque se incluyen algunas entrevistas realizadas a referentes del mercado inmobiliario y documentos estadísticos que dan información sobre la construcción.

2 En Barrancabermeja, se instaló la refinería más importante de Colombia. Su construcción fue una de las pocas exigencias que se le hizo a la Tropical Oil Company, y, desde su inauguración, en la segunda década del siglo pasado, hasta hoy, lidera la actividad en el país. En Comodoro Rivadavia, en cambio, la actividad de la industria petrolera se concentra principalmente en la etapa de extracción.

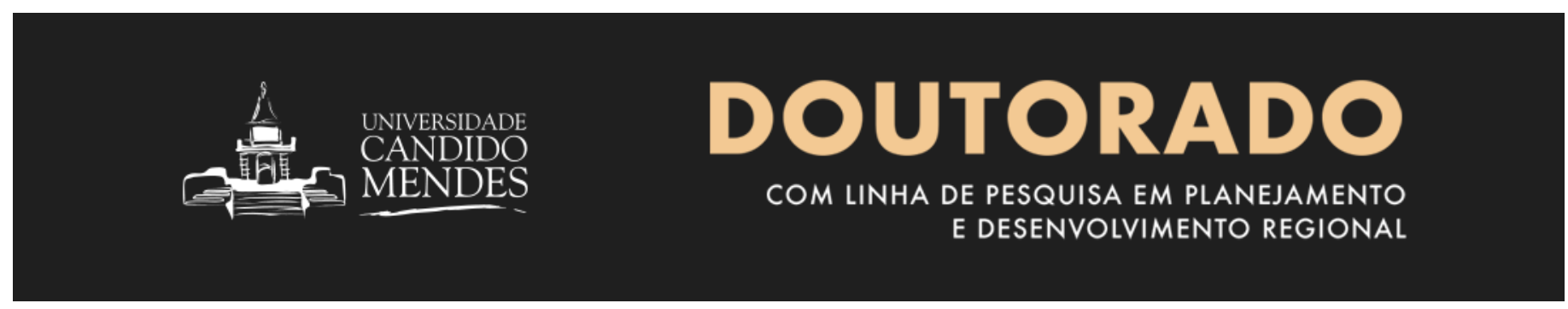




\section{Espacio y petróleo}

La ciudad capitalista puede ser definida en una doble relación productor-producto (Santos, 1998). Es, por un lado, el marco de la ganancia y la condición esencial para la producción y la reproducción social; pero, al mismo tiempo, los elementos que la componen son producidos para obtener ganancia (Topalov, 1979). La construcción tiene algunas características particulares: 1. El suelo (soporte de la producción inmobiliaria) es único e irreproducible; por tanto, su uso implica su agotamiento y, para cada nueva producción, se necesita un nuevo suelo (Topalov, 1979); 2. El tiempo de producción es muy largo, con una rotación de capital muy prolongada por el tiempo que se demora la construcción y la circulación.

Por estas razones, factores ajenos a la actividad como fluctuaciones en la mano de obra, en la demanda o en el capital en circulación pueden cambiar las condiciones, y "siendo su reacción más lenta que en otras esferas el efecto de la contracción es más severo, pues eventualmente deja como productos inacabados y, por lo tanto, no realizables, mayores porciones de valor" (Jaramillo 2009, 102). Lo anterior reafirma la característica cíclica de la construcción por la sensibilidad a los cambios, que puede ser visible en la evolución de los permisos de construcción más que en los precios (Baer y Kaum, 2013).

En relación con el precio del suelo urbano, Jaramillo (2009) distingue tres tipos de cambio: los movimientos estructurales generales, relacionados con una tendencia en el largo plazo al alza; los movimientos coyunturales generales, que explican algunas de las fluctuaciones; y los movimientos estructurales particulares, atados a cambios en los usos y densidades permitidas. En las ciudades productoras de petróleo, las variaciones del precio en el mercado internacional regulado de petróleo y los ciclos expansivos y regresivos de la industria tienen consecuencias importantes sobre los mercados locales desregulados, movimientos que podrían catalogarse dentro de los movimientos coyunturales generales, explicando algunas de las subas y bajas del precio dentro de esa tendencia general al alza.

La mayor parte del espacio construido está dedicado a la vivienda. Esta es una de las necesidades elementales del ser humano. Su acceso en el sistema capitalista se da en el mercado. Aunque es una mercancía que se compra y se vende, sin embargo, tiene algunas particularidades. En primer lugar, "a diferencia de los mercados de acciones, bonos y materias primas de todo el mundo, los merca- dos de tierra están desorganizados" (Dowall, 1993:24)3. Además, como el suelo que la soporta es único e irreproducible, el valor de cada porción se distribuye desigualmente: "cada lugar concreto en una ciudad, y por lo tanto, cada lote de terreno tiene potencialidades diversas de sostener procesos de consumo de espacio construido" (Jaramillo, 2009:117). El espacio, entonces, no es un continuo homogéneo, la división social del espacio está relacionada como la división de clase y la estructura misma del espacio.

Para acceder a la vivienda, en términos generales, "las tres alternativas básicas que tiene la población para acceder a una vivienda son: a) el alquiler; b) el crédito hipotecario; y c) la autoproducción-autofinanciamiento" (Coulomb, 2006:126). En América Latina, la dualidad y persistencia de la informalidad y formalidad son caras de la misma moneda. En la región, se pueden observar frecuentemente variaciones autogestivas $y / 0$ informales: "la primera no involucra ninguna operación de financiamiento y se puede manifestar, ya sea bajo la forma de ocupación, o de transferencia de propiedad o derechos de concesión de uso (...) la segunda de estas formas de acceso involucra financiamiento, pero este es intermediado por el Estado y presenta la particularidad de concesión de subsidios en la atribución de lotes urbanos" (Abramo, 2009:87).

\section{El mercado inmobiliario de Barrancabermeja y Comodoro Rivadavia en el siglo XXI}

En Barrancabermeja en el año 2010, se anunció la ejecución del Plan de Modernización de la Refinería de Barrancabermeja (PMRB). Se presupuestó que las obras programadas demandarían una inversión aproximada de entre cuatro y seis mil millones de dólares y la llegada de al menos cinco mil trabajadores a la ciudad. Más allá de las mejoras técnicas en las diversas plantas y procesos de refinación, el PMRB fue un punto de quiebre en el mercado inmobiliario local. Por la expectativa que generó y el inicio de los primeros trabajos -que efectivamente impulsaron la economía de la ciudad e incrementaron la llegada de población- se produjo un aumento en la construcción de viviendas. Las declaraciones oficiales alentaron las expectativas:

"se decía que debíamos prepararnos, en declaraciones que hizo la cámara de comercio en algún

3 Traducción propia. 
momento, el presidente de Ecopetrol a través de un foro que hubo en la cámara de comercio, donde invitaba a los empresarios de Barranca, a la sociedad de Barranca a prepararse estructuralmente para acoger a más de 5.000 personas que llegaban a Barranca a desarrollar el proyecto de la refinería que iba a demorar cinco años" (Moreno, entrevista, 19 de enero de 2019).

Hasta ese momento, el sector inmobiliario formal había tenido varios años de estancamiento. En la ciudad, se construía poco. Además de las difíciles condiciones climáticas -con temperaturas promedio que superan los 30 grados centígrados todo el año- se sumó el conflicto armado. Barrancabermeja se transformó en un territorio violento con "una tasa de mortalidad per cápita que en el año 2000 fue tres veces más alta que en el resto del país" (Gills, 2009:17).

"por los años 90 debido al proceso de violencia que se generaba acá por la zona tan cercana de conflicto, y ser una zona de influencia guerrillera, y después paramilitar los precios de arrendamiento y de venta estaban completamente castigados" (Ogliastri, entrevista, 21 de enero de 2019).

Con los precios "castigados", las inversiones inmobiliarias eran escasas. La falta de dinamismo en el sector formal contrastaba con el intenso crecimiento de la ciudad cruzando el puente y la línea del ferrocarril para ese momento en desuso. Según Gills (2009): "los refugiados inundaron Barrancabermeja y la ciudad se llenó de víctimas desplazadas de la violencia rural. La mayoría de los refugiados vinieron de áreas que una vez estuvieron bajo el control de la guerrilla" (p.316). Como se observa en el siguiente mapa, la ciudad de origen informal constituye alrededor del $50 \%$ de la mancha urbana de Barrancabermeja. En este caso, la llegada de migrantes no resultó en un aumento de los precios. La pobreza y la necesidad, además de la falta de atención en la ciudad receptora, los forzó a establecerse donde y como pudieran. Tenemos que pensar que, a diferencia de otros, los movimientos migratorios, estos desplazamientos forzados no tienen un plan ni una decisión. Muchos tenían poco, y llegaron con nada.

\section{Plano 1. Los tres sectores de la ciudad}

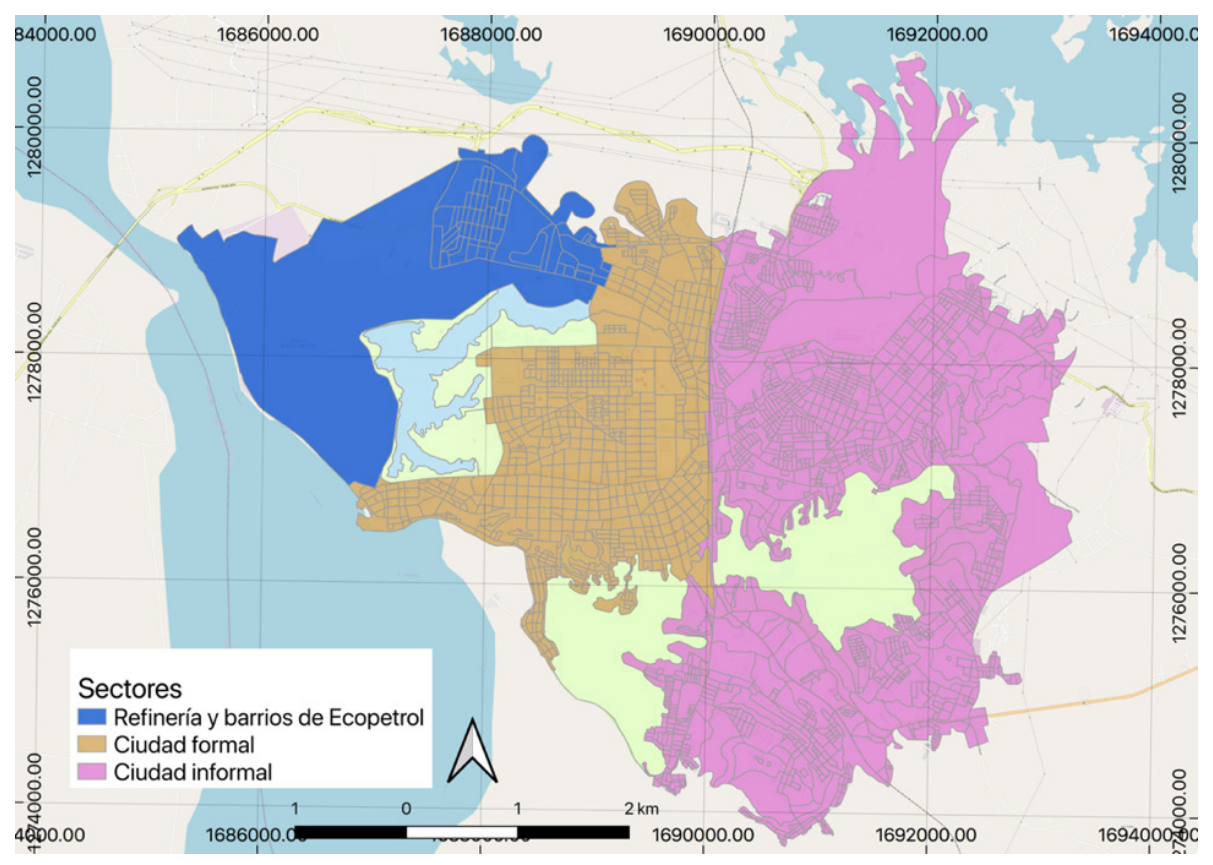

Fuente: Elaboración propia con QGIS.

En el plano anterior, se observa claramente la relevancia de la construcción informal y el avance de la toma de tierras en la estructura urbana de la ciudad; al mismo tiempo, refleja fielmente la tajante división. La línea del ferrocarril atraviesa la ciudad de norte a sur justo entre el color naranja y el rosa. Se observan los barrios y la 
refinería en azul.

Sin embargo, la expectativa generada por el anuncio de la modernización de la refinería transformó la situación. La construcción en la ciudad creció y alcanzó niveles inusitados. El gráfico que se presenta a continuación da cuenta de ese proceso. Entre 2005 y 2009, los metros cuadrados construidos para vivienda en Barrancabermeja no superaban la barrera de los 50.000, entre 2010 y 2014, en cambio, alcanzaron los 200.000 metros cuadrados, cuadruplicando la cantidad.

Después de 2015, el PMRB tambaleó hasta ser finalmente cancelado, desencadenando una crisis en el sector inmobiliario visible en la inmensa cantidad de construcciones sin terminar y el desplome de las operaciones de compra y alquiler ante una tardía respuesta de los precios que se negaban a bajar y el desplome de la demanda 4 .

$4 \quad$ Entre los factores que llevaron a la suspensión estaban: incumplimientos en las licencias ambientales, reubicación de los sectores aledaños a la refinería, como Termogalán-Berlín, invalidad del proyecto, caído del precio internacional del petróleo, escándalos sobre los sobrecostos de las obras de ampliación de la refinería de Cartagena, entre otras justificaciones del Gobierno y la estatal.

\section{Gráfico 1. Área construida en metros cuadrados para vivienda, 2005-2016}

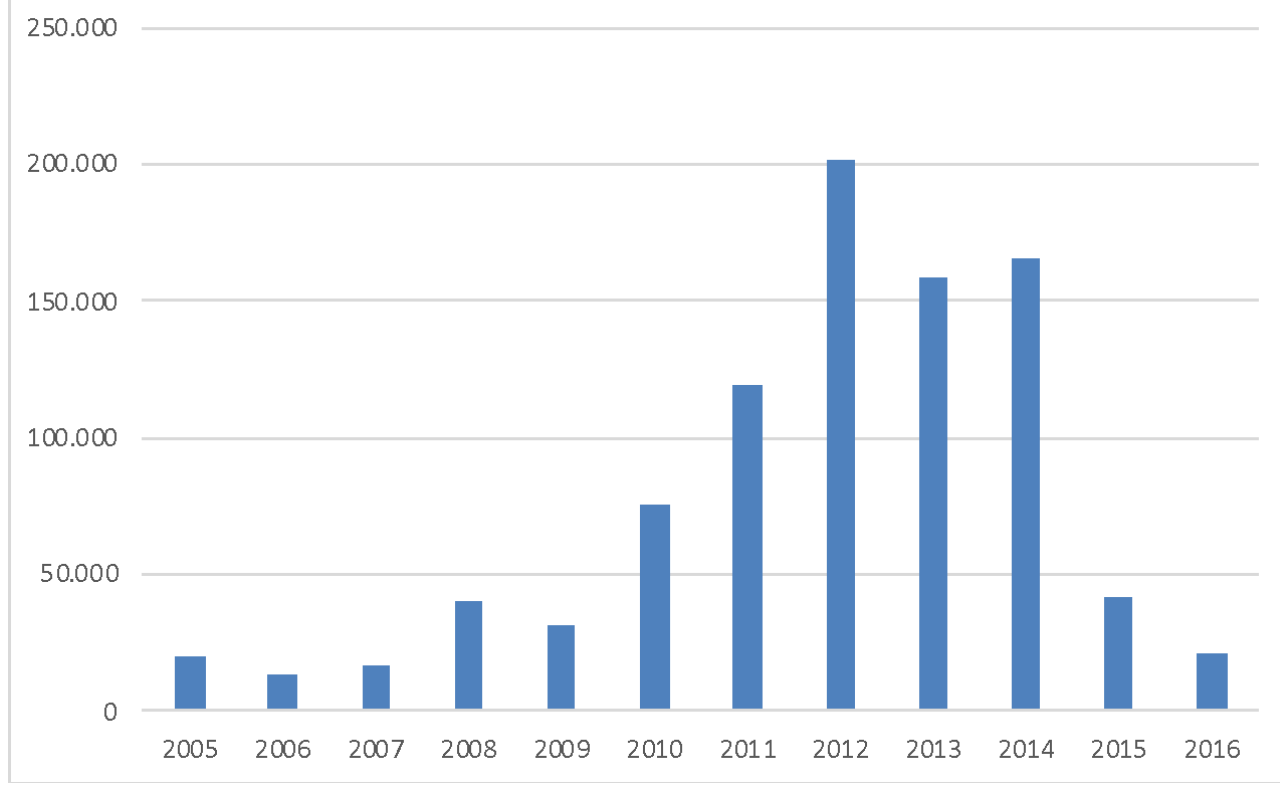

Fuente: Elaboración propia según DANE (2019).

La construcción de vivienda nueva en Barrancabermeja tuvo varios actores. Por un lado, arribaron empresas de alcance nacional y regional que levantaron torres de departamentos en varias zonas de la ciudad cambiando por completo el paisaje de casas de una y dos plantas que había caracterizado hasta entonces a la ciudad; por otro lado, se registró una fuerte participación de personas que buscaron obtener una parte del excedente de la renta petrolera refaccionando o construyendo en sus casas habitaciones y departamentos para alquilar.

"todo el mundo se abalanzó a construir, todo el mundo se abalanzó a remodelar sus viviendas de $300 \mathrm{~m}$ para convertirlas en dos casas de $150 \mathrm{~m}$, o en edificios de cinco pisos en un área de 300 m (...) personas independientes, profesionales independientes sacaron muchos créditos. El movimiento financiero también se disparó en el tema de préstamos para construcción" (Moreno, entrevista, 21 de enero de 2019).

En Comodoro Rivadavia, como vimos antes, el territorio se había fragmentado en varios Company Towns o pueblos autónomos en los cuales la empresa, además 
de empleadora, ejercía también como terrateniente. La integración de esos territorios en el ejido municipal y su transformación en barrios generó una ciudad fragmentada espacial y socialmente. La historia urbana de cada uno de esos fragmentos además da cuenta de los distintos tipos de acceso a la vivienda (Ruiz, Baeza y Arce, 2014). En primer lugar, la zona sur, que históricamente asumió un rol comercial y administrativo complementario a aquellos territorios con una función productiva única instalada en los campamentos petroleros aledaños. Su crecimiento estuvo marcado por el avance de la toma de tierras (Vazquez, 2019; Bachiller, 2015). En segundo lugar, los barrios del norte, que se configuraron alrededor de los pozos de extracción y se integraron tardíamente al ejido municipal, construidos y planeados por las empresas, tampoco bien conectados entre ellos. Por último, Rada
Tilly, que, aunque formalmente una ciudad autónoma, en la práctica, se constituyó como un barrio residencial para las capas medias y altas de la población comodorense. Es de perentorio menester aclarar que, aunque las agrupamos con fines analíticos, existen grandes diferencias al interior de cada uno de estos tres sectores. Particularmente en la zona sur de la ciudad, podemos diferenciar el centro de los barrios nacidos informalmente, que ampliaron el ejido hacia el sur oeste de la ciudad, y barrios como Roca y Pueyrredón, que están muy relacionados a estratos de mayor ingreso y se consolidaron hacia los años ochenta (Ruiz, Baeza y arce, 2014). Los agrupamos, porque los límites entre estos son más difusos, y la trama urbana presenta continuidad.

\section{Mapa 2. Los sectores de Comodoro Rivadavia}

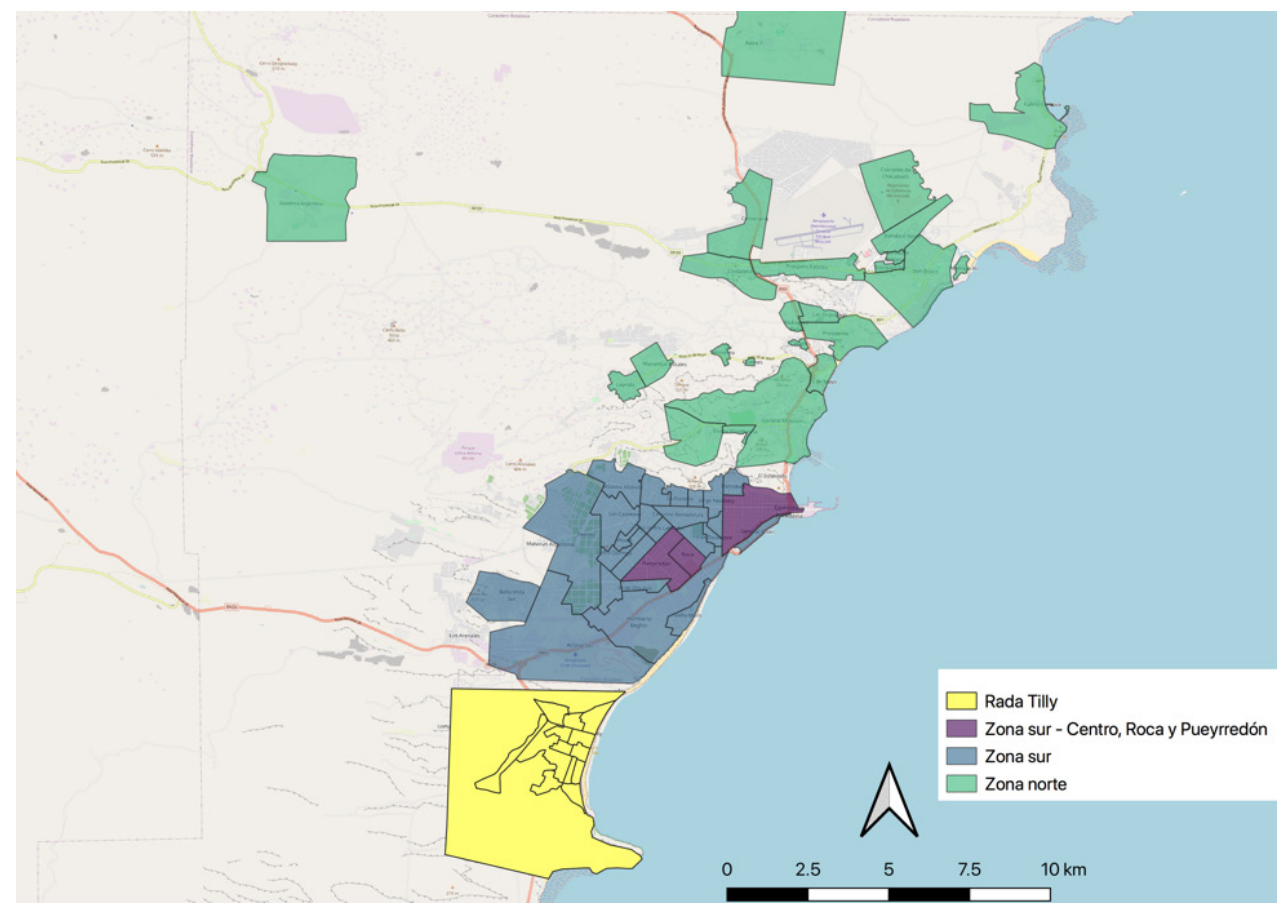

Fuente: Elaboración propia con base en labSIGyT de la UNP, 2018

Varios trabajos dan cuenta de las percepciones y definiciones que tienen los habitantes de los distintos sectores de la ciudad. Las categorías de diferenciación pasan por la relación con la industria petrolera con la denominación de ypefeanos, pero alcanza otras características como las recurrentes denominaciones a los nacidos y criados (NYC), que, según Grimson y Baeza (2016:4), son: "una categoría central de la vida social, que presupone que hay o debería haber ciertos privilegios para aquellos que son verdaderamente del lugar". En estas ciudades, como consecuencia de las migraciones temporales en momentos de auge económico, crece la idea del migrante como una persona que se aprovecha de la situación y abandona la ciudad ante un escenario de crisis: "una de las claves para entender ese rechazo al foráneo está en el imaginario que sostiene que llegan a la ciudad a "hacer plata" y luego se van (porque "tienen las valijas listas atrás de la puerta") (Barrionuevo, 2019:76). 
En ese sentido, la privatización de Yacimientos Petrolíferos Fiscales tuvo profundas repercusiones. Generó el "achicamiento de los planteles de empleados, tercerizaciones de actividades de apoyo a las empresas, debilitamiento del poder de negociación sindical" (Torres, 2012:286) y el desmantelamiento de la contención que ofrecía YPF a sus trabajadores. Sin embargo, la producción se recuperó rápidamente, aunque sobre las bases de exploraciones anteriores. En 2004, se inició un momento de auge denominado el segundo boom petrolero: "el cambio en las condiciones económicas nacionales a partir de la salida de la convertibilidad en 2002 creó una coyuntura favorable en términos cambiarios para la explotación de los commodities. El petróleo, actividad extractiva y exportable, registró un ciclo de expansión en la explotación entre 2004 y 2008" (Barrionuevo, 2019:56).

La construcción en Comodoro Rivadavia, a diferencia de Barrancabermeja, no parece haber tenido un pico durante esas décadas, aunque tampoco alcanzó niveles tan bajos como los registrados en el caso colombiano. Hasta el 2005, creció coincidiendo con la salida de la crisis de 2001 y el boom hasta 2008. En el periodo analizado, la mayor cantidad de metros cuadrados cubiertos construidos se registró entre 2014 y 2018, con un marcado descenso hacia 2019. Al igual que Barrancabermeja, la construcción fue impulsada, en gran parte, por inversores locales, que buscaban obtener de esta manera excedentes de la renta petrolera (Peters, 2016).

"mucha gente que no era propia sector inmobiliario y constructivo, mucha gente fuera del sector se dio a construir. Por decirte, un medico, un abogado, o gente que tenía acceso a dinero, vio en el negocio inmobiliario una veta e ingresaron" (Entrevista 2).

La construcción formal se concentró en zonas específlcas. Parece existir un "acuerdo unánime sostiene que en la ciudad "hay pocos lugares buenos para construir" y todos apuntan a las mismas áreas: Rada Tilly, el centro, kilómetro 3, los barrios Roca y Pueyrredón; en contadas ocasiones se menciona algún que otro barrio como La Loma, Diadema, o el Km 8" (Bachiller, 2018:125). El tipo de oferta, como señaló un referente del mercado inmobiliario de la ciudad, se adaptó a la demanda de migrantes que llegan solos, con construcciones de departamentos de un dormitorio porque "achicás el tique de entrada de valor de venta y en los momentos de auge se alquilan como pan caliente" (Entrevista 1).

\section{Gráfico 2. Área cubierta construida en Comodoro Rivadavia, 2005-2019}

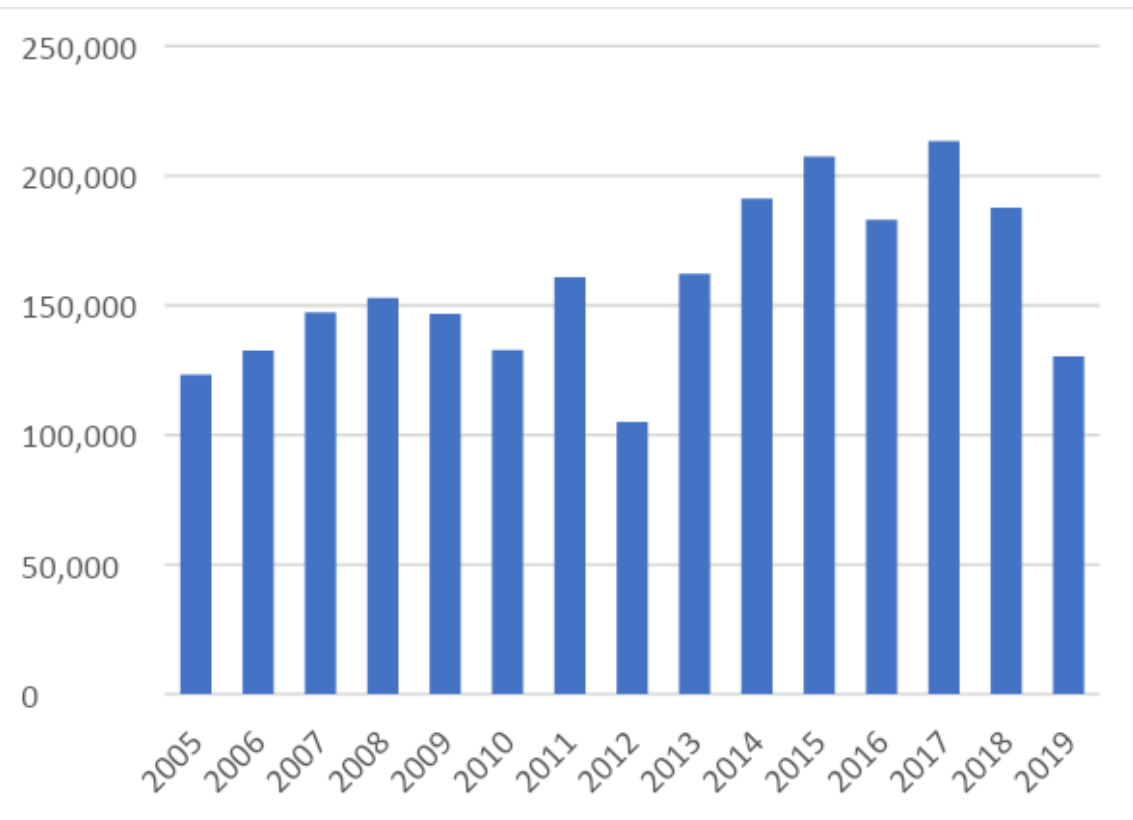

Fuente: Elaboración propia con base en datos de la Dirección General de Estadísticas y Censos de Chubut. 
Por supuesto, los datos dan cuenta del área construida con permiso y, por tanto, deja por fuera las tomas de tierras y las construcciones informales. En Comodoro Rivadavia, el denominado segundo boom petrolero tuvo consecuencias demográficas y espaciales notorias. Bachiller estudió el impacto del crecimiento de la explotación, la llegada de migrantes y las tomas de tierras en el mercado inmobiliario y en la configuración socioespacial actual de la ciudad. Según él, "la mayor parte de los actuales barrios populares, especialmente en la zona sur y oeste, crecieron mediante las dinámicas de autopromoción de la vivienda y la toma de tierras. Tal es así que hoy en día, el 75\% de la población reside en dicha área (latasa, 2011)" (Bachiller, etnografiando:3).

A diferencia del caso colombiano, en el que la mayor parte de la población que arribó porque fue obligada violentamente a dejar su lugar de origen, en Comodoro Rivadavia, las tomas de tierras no se limitan a personas pobres y sin recursos. La escasez de tierras urbanizables (ya sea por estar reservadas para la actividad hidrocarburífera, por dificultades topográficas o por la existencia de pasivos ambientales) genera que familias con recursos disponibles tengan que recurrir al acceso informal del suelo. A diferencia de otras tomas, en este caso, se evidencian construcciones y mejoras rápidamente, indicando que no es solamente un problema de dinero sino de disponibilidad de suelo y del elevado precio del suelo efectivamente disponible.

En Comodoro Rivadavia, "la industria petrolera ha impedido la utilización satisfactoria de distintos espacios locales (...) encontramos áreas urbanizadas sobre antiguos yacimientos petroleros, los casos más notorios se dan en zona Norte, donde se han levantado barrios enteros sobre suelos inestables y mal sellados" (Freddo, 2013:6). Bachiller, por su parte, da cuenta del problema que genera en términos ambientales y urbanísticos la ubicación de los pozos: "entre los empresarios existe un amplio consenso acerca de que la topografía y el pasivo ambiental constituyen fuertes límites en las posibilidades de expansión del mercado inmobiliario" (Bachiller et al., 2020:14).

\section{Mapa 3. Ubicación de los pozos en Comodoro Rivadavia}

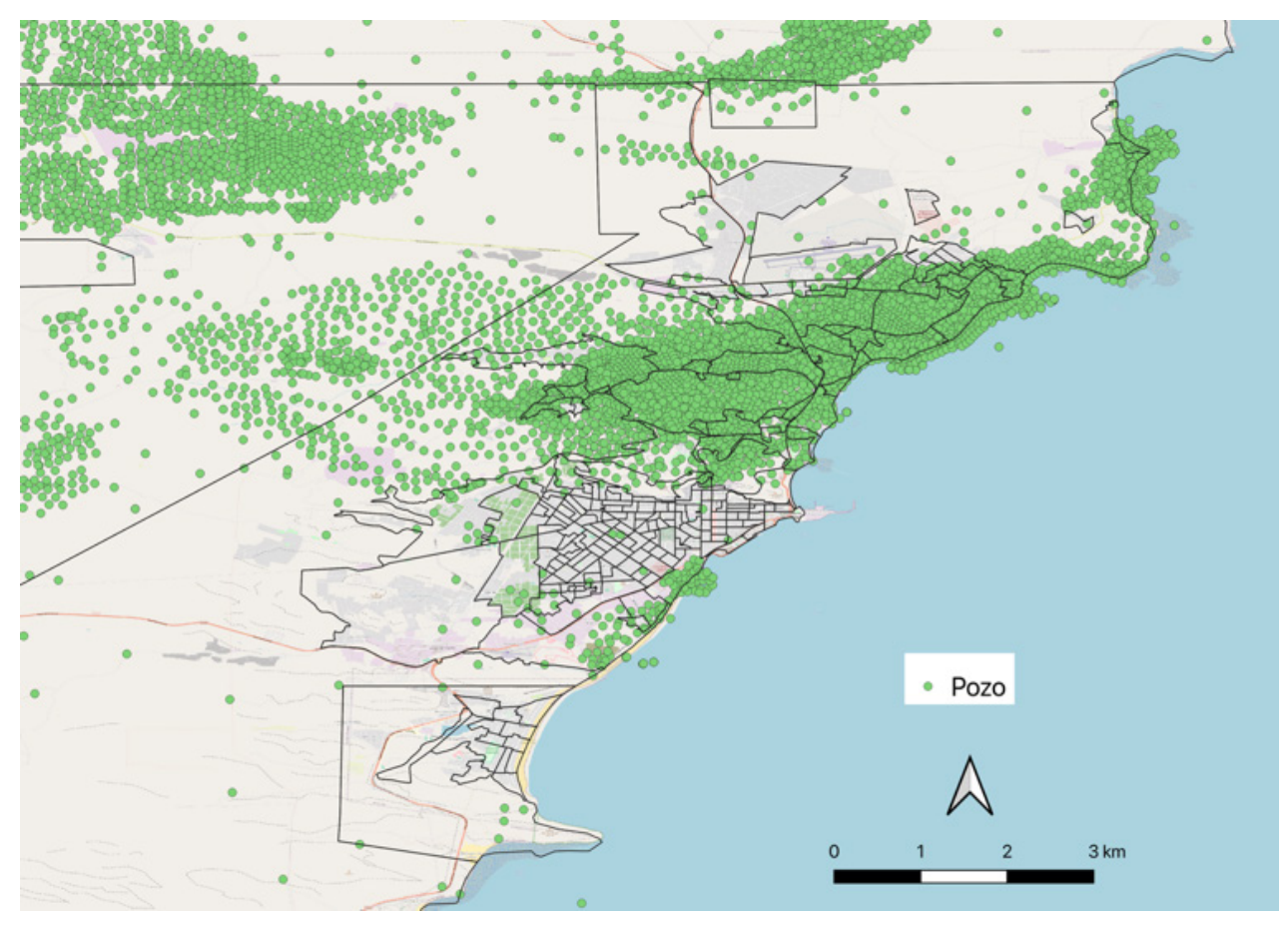

Fuente: Elaboración propia con base en https://www.ign.gob.ar/ NuestrasActividades/InformacionGeoespacial/CapasSIG

En el caso colombiano, los pozos se encuentran ubicados mayoritariamente afuera del área urbana, aunque representan un problema para la zona rural del municipio, que también convive entre pozos, máquinas extractoras y oleoductos. Sin embargo, la industria petrolera tiene serias repercusiones ambientales que afectan la vida 
en la ciudad. Por un lado, la ubicación de la refinería en el corazón comercial de la ciudad generó la contaminación de los cuerpos hídricos. Por otro, la intensa actividad de las antorchas de quemado deja olores desagradables y serias dudas sobre la calidad del aire que se respira en Barrancabermeja.

\section{Mapa 4. Ubicación de los pozos petroleros en Barrancabermeja}

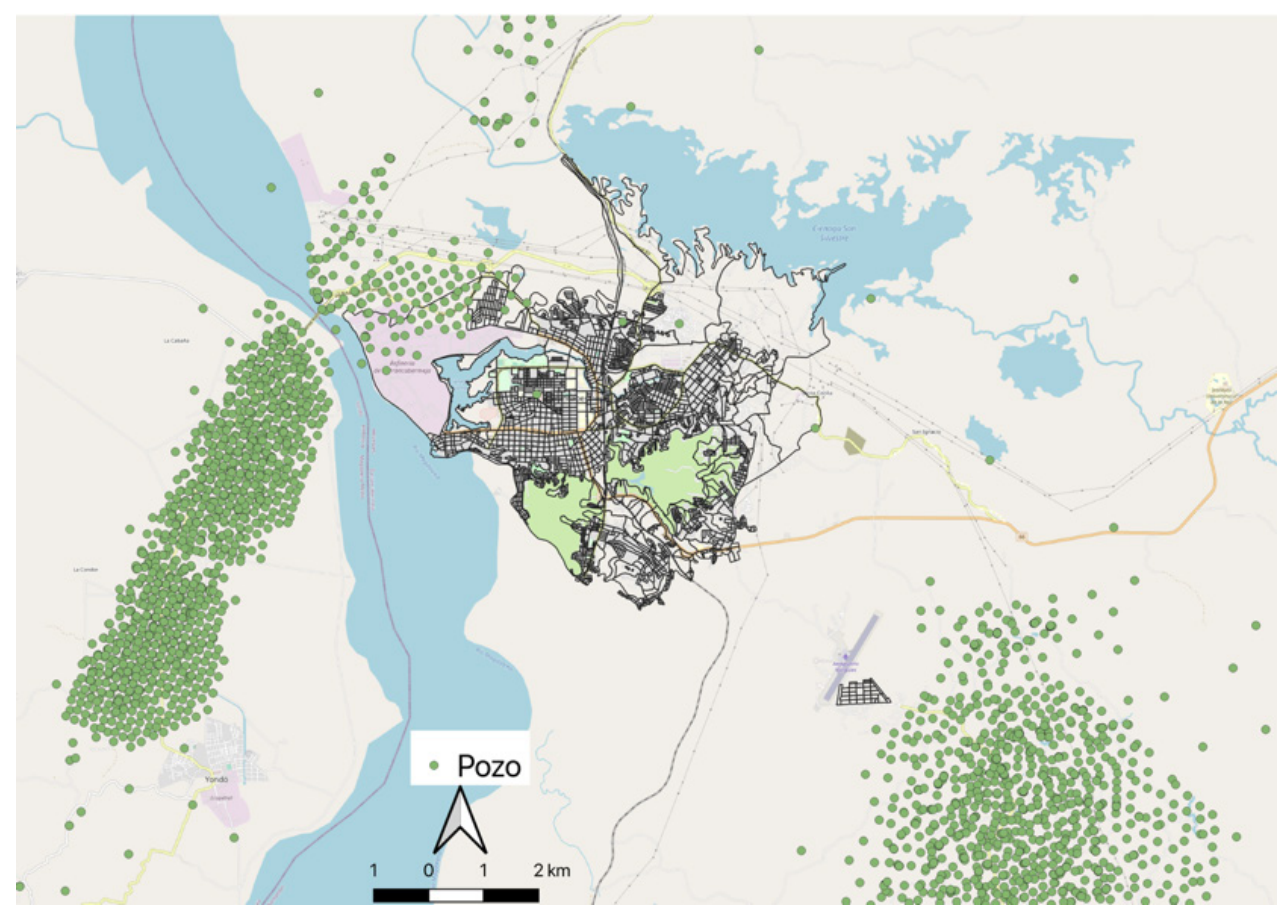

Fuente: Elaboración propia con base en ANH, https://geovisor.anh.gov.co/tierras/\#

\section{Conclusiones}

Barrancabermeja y Comodoro Rivadavia tienen una relación con la industria petrolera desde su establecimiento a inicios del siglo XX. El precio regulado en el mercado internacional del barril de petróleo y los ciclos expansivos y regresivos de la industria tienen consecuencias directas sobre la economía, la sociedad y el territorio en las dos ciudades. En el siglo XXI, la intensa variación del precio del barril de petróleo en el mercado internacional tuvo consecuencias notorias.

En Barrancabermeja, se programó la modernización de la refinería de la ciudad. Un plan que requeriría una inversión multimillonaria y que demandaría la llegada de al menos cinco mil trabajadores, según fuentes oficiales. Las expectativas generadas por el PMRB, confirmadas por el inicio de algunas obras previas y las voces oficiales, generaron el aumento de la especulación sobre el precio del suelo y los inmuebles. Entre 2010 y 2014, el número de metros cuadrados construidos para vivienda se cuadruplicó. Sin embargo, el derrumbe del petróleo y distintos obstáculos políticos impidieron el desarrollo del proyecto y las perspectivas se desplomaron.

En Comodoro Rivadavia, la privatización de YPF había significado un momento de crisis económica y simbólica con el desmonte de la contención que ofrecía la empresa. Sin embargo, la salida de la convertibilidad y el aumento del precio del barril impulsaron la extracción de petróleo en la ciudad. Un nuevo boom petrolero tuvo lugar y generó importantes cambios socioespaciales. La construcción creció, aunque de forma más moderada que en el caso colombiano. Las nuevas obras se ajustaron a la migración que llegaba: monoambientes para los trabajadores que arribaban a la ciudad. Por otra parte, el aumento de los precios y de la población presionaron sobre un escaso parque inmobiliario, dejando en evidencia una vez más la relación entre auge 
económico y toma de tierras.

En los dos casos, en la construcción, se observó una participación de personas "ajenas al sector", que buscaron apropiarse una parte de la renta petrolera mediante el alquiler y venta de inmuebles. Además del aumento del precio a causa de los altos salarios y la especulación, la industria petrolera y sus pasivos ambientales deja fuera de circulación, particularmente en Comodoro Rivadavia, extensos terrenos, limitando la expansión de la ciudad. En resumen, el mercado inmobiliario de las ciudades petroleras está fuertemente vinculado a los movimientos de la industria petrolera, compartiendo las expectativas de crecimiento y los momentos de crisis.

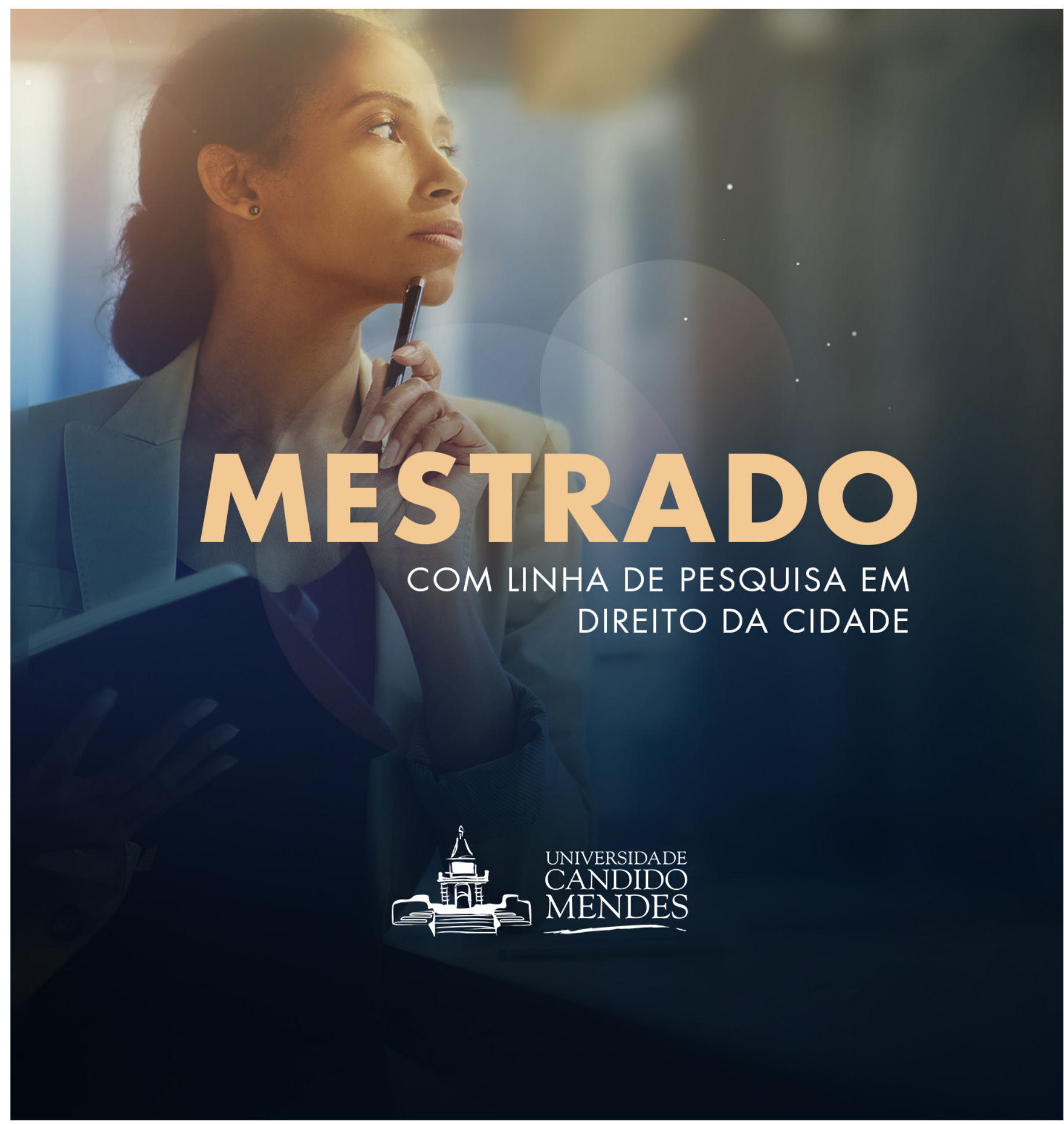




\section{Referências}

Arroyo, Andrés y Cosío, Fernando (2015), Impacto fiscal de la volatilidad del precio del petróleo en América Latina y el Caribe: estudio sobre las causas y las consecuencias de la caída de los precios del petróleo y análisis de opciones de política para encaminar sus impactos, Documentos de ProyectosNo.680, Santiago de Chile, CEPAL.

Arias Trujillo, Ricardo (2010), Historia contemporánea de Colombia (1920-2010), Bogotá, Universidad de los Andes.

Baer, Luis; Kauw, Mark (2013), "Mercado inmobiliario y acceso a la vivienda formal en la Ciudad de Buenos Aires, y su contexto metropolitano, entre 2003 y 2013" en: Revista EURE, VOL 42 N.126.

Bachiller, Santiago; Natalia Usach, Magalí Chanampa y Bianca Freddo (2020) "Funcionamiento del mercado inmobiliario en una sociedad petrolera: condicionamiento para los sectores populares". En: Revista Vivienda y Ciudad. ISSN: 2422-670X. En prensa.

Bachiller, Santiago (2015). Etnografiando las últimas tierras en Comodoro Rivadavia. En: XI Jornadas de Sociología. Facultad de Ciencias Sociales, Universidad de Buenos Aires, Buenos Aires

Bachiller, Santiago. (2018). Petróleo, planificación urbana y exclusión residencial en Comodoro Rivadavia. Identidades, 14(8), pp. 119-137. Recuperado de https://iidentidadess.wordpress.com/numeros-anteriores/numero-14/

Barrionuevo, Natalia (2019). (Re)producción y legitimación de fronteras sociales "establecidas" a partir del segundo boom petrolero (2004-2014) en Comodoro Rivadavia. Tesis para obtener el título de doctor en sociología, Universidad Nacional de San Martín.

Buenahora, Gonzalo (1982). Sangre y petróleo. S.E: Bogotá.

DANE (Departamento Administrativo Nacional de Estadística) (2019), Estadísticas de Licencias de Construcción. Bogotá, DANE [EXCEL] Disponible en: https://www.dane.gov.co/files/investigaciones/construccion/lic_construccion/2019/serie_tipo_base_municipios_dic19.xlsx

Freddo, Bianca (2013). "Perspectivas de análisis para estudiar la ciudad. Estudio de caso: reflexiones sobre los proyectos para mejorar la trama urbana de la ciudad de Comodoro Rivadavia". Observatorio de la Economía Latinoamericana No 187. Bajado de: [http://www.eumed.net/cursecon/ecolat/ar/2013/ ordenanzas.html].

Grimson, Alejandro y Baeza, Brígida (2016). "Desacoples entre el nivel de ingresos y jerarquías simbólicas en Comodoro Rivadavia. Acerca de las legitimidades de las desigualdades sociales". Disponible en: https://iidentidadess.files.wordpress.com/2015/07/01-identidades-10-6-2016-grimson-baeza.pdf

Gill, Leslie (2009), "The Parastate in Colombia: Political Violence and the Restructuring of Barrancabermeja”, Anthropologica, Toronto, University of Toronto y Canandian Anthropology Society, Vol. 51, No. 2, pp. 313-325.

Jaramillo, Samuel (1981). Crisis de los medios de Consumo Colectivo Urbano y Capitalismo periférico. En: Revista Desarrollo y Sociedad.

Palacios, Marco (1995). Entre la legitimidad y la violencia: Colombia, 1875-1994. Norma: Bogotá.

Peters, Stefan (2016). Petróleo, política y sociedad en Chubut. Reflexiones teórico-conceptuales en torno al rentismo. En: Revista Identidades, dossier 3 , año 6.

Topalov, Christian (1979). La urbanización capitalista: algunos elementos para su análisis. edicol: México.

Torres, Fernanda (2012). La privatización de YPF en Comodoro Rivadavia. Algunas características y consecuencias sociales y laborales. En: Revista trabajo y sociedad, N.18, Vol.XV.

Usach, Natalia y Freddo, Bianca (2014). "Crecimiento de una ciudad dispersa: análisis y reflexiones del caso de la ciudad de Comodoro Rivadavia". IX Jornadas Patagónicas de Geografía. UNPA, Santa Cruz,

Vázquez, Letizia (2019). Boom petrolero, crecimiento demográfico y expansión urbana en Comodoro Rivadavia (1958-1963). En: Revista Pilquen, vol 2 n.4, pp.1-11. 